\title{
ENDOMORPHISM RINGS AND DIRECT SUMS OF TORSION FREE ABELIAN GROUPS
}

\author{
BY
}

\author{
D. M. ARNOLD(1) AND E. L. LADY
}

\begin{abstract}
Properties of abelian groups related to a given finite rank torsion free abelian group $A$ are analyzed in terms of End $(A)$, the endomorphism ring of $A$. This point of view gives rise to generalizations of some classical theorems by $\mathrm{R}$. Baer and examples of pathological direct sum decompositions of finite rank torsion free abelian groups.
\end{abstract}

An abelian group $G$ is $A \cdot$ free if $G$ is isomorphic to a direct sum of copies of an abelian group $A$ and $A$-projective if $G$ is a direct summand of an $A$-free group. Define $S_{A}(G)$ to be the subgroup of $G$ generated by $\{f(A) \mid f$ $\left.\epsilon \operatorname{Hom}_{Z}(A, G)\right\}$.

The following properties are considered for a finite rank torsion free abelian group $A$.

(I) Every $A$-projective group is $A$-free.

(II) Every exact sequence $0 \rightarrow B \rightarrow G \rightarrow C \rightarrow 0$ of abelian groups, where $S_{A}(G)+B=G$ and $C$ is $A$-projective, is split exact.

(III) Every subgroup $B$ of an $A$-free group with $S_{A}(B)=B$ is $A$-free.

(IV) Every subgroup $B$ of an $A$-projective group with $S_{A}(B)=B$ is $A$ projective.

Theorem 1. (a) (I) is true iff every projective right End (A)-module is free.

(b) (II) is true iff $I A \neq A$ for all proper right ideals $I$ of End $(A)$.

(c) (II) and (III) are true iff End ( $A$ ) is right principal and has no zerodivisors.

(d) (II) and (IV) are true iff End ( $A$ ) is right hereditary.

Conditions (I)-(IV) are all true if End $(A)$ is a principal ideal domain. Examples of groups with this property include:

Received by the editors January 11,1974 .

AMS (MOS) subject classifications (1970). Primary 20K15.

Key words and phrases. Strongly indecomposable groups, Krull-Schmidt property, quasi-isomorphism.

(1) Preparation of manuscript supported, in part, by NSF Grant GP-40872. 
(i) $\operatorname{rank}(A)=1$;

(ii) $\operatorname{rank}(A)=2$ and the typeset of $A$ has more than 3 elements (Beaumont-Pierce [4]);

(iii) $A$ is indecomposable of finite rank and $Z / p Z$-dimension $(A / p A) \leq$ 1 for all primes $p$ (Murley [13]); and

(iv) rigid groups (Fuchs [9, p. 124]).

Suppose that $I$ is a right ideal of $\operatorname{End}(A)$ with $I A=A$. If $\operatorname{End}(A)$ is commutative, right principal, or right hereditary then $I=$ End $(A)$. Furthermore, if $A$ is strongly indecomposable (i.e., $n A \subset B \oplus C \subset A$ for some $0 \neq n \in Z$ implies that $B=0$ or $C=0$ ) or $Q \otimes_{Z}$ End $(A)$ is semisimple then End $(A) / I$ is finite. On the other hand, there does exist a finite rank torsion free abelian group $A$ and a two-sided ideal $I$ of End $(A)$ with $I A=A$ and $\operatorname{End}(A) / I$ infinite.

$\$ 4$ includes some cancellation and exchange properties for certain classes of finite rank torsion free abelian groups. Most notably, we have

Theorem 2. (a) If $A \oplus H_{1} \simeq A \oplus H$, and if $A$ and $H$ have no quasi-summands in common then $H \simeq H_{1}$.

(b) If $A \oplus K=B \oplus C$, where $A$ and $K$ have no quasi-summands in common, then $A \oplus K=B_{1} \oplus C_{1} \oplus K$ for some $B_{1} \subset B, C_{1} \subset C$.

Finally, properties (I)-(IV) are examined, for the case that $\operatorname{rank}(A)=2$, in $\$ 5$.

Basic references are Fuchs [8] and [0] for general properties of abelian groups; Reid [15] for quasi-endomorphisms and quasi-decompositions of torsion free abelian groups; and Lambek [12] or Bass [3] for general properties of rings and modules.

1. A-projective groups. The following general homological remarks are essential to the proofs in this section. Let $\varrho$ be the category of abelian groups, $A$ an abelian group, $R$ the endomorphism ring of $A$ and $\Re_{R}$ the category of right $R$-modules. A left exact functor $H: \mathcal{G} \rightarrow \mathbb{M}_{R}$ is defined by letting $H(G)=\operatorname{Hom}_{Z}(A, G)$ and $H(f)(g)=f g$ where $f: G \rightarrow G^{\prime}$ is an abelian group homomorphism. Moreover, there is a right exact functor $T: \pi_{R} \rightarrow \varrho$ given by $T(M)=M \otimes_{R} A$, regarding $A$ as a left $R$-module, and $T(f)=f \otimes 1$ for a homomorphism $f: M \rightarrow M^{\prime}$ of right $R$-modules.

There is a natural transformation $\theta$ from $T H$ to the identity functor on G, where $\theta_{G}: \operatorname{Hom}_{Z}(A, G) \otimes_{R} A \rightarrow G$ is defined by $\theta_{G}(f \otimes a)=f(a)$ for $a \epsilon$ $A, f \in \operatorname{Hom}_{Z}(A, G)$, and $G$ an abelian group. On the other hand, there is a natural transformation $\phi$ from the identity functor on $\pi_{R}$ to $H T$ where 
$\phi_{M}: M \rightarrow \operatorname{Hom}_{Z}\left(A, M \otimes_{R} A\right)$ is given by letting $\phi_{M}(x)(a)=x \otimes a$. (These functors and transformations are analogous to those used in defining a Morita equivalence, e.g., see Bass [3].)

Theorem 1.1. Let $A$ be a torsion free abelian group of finite rank. The functor $H$ from the category of A-projective groups to the category of projective right End $(A)$-modules is a category equivalence with inverse $T$.

Proof. Let $\mathcal{P}_{A}$ be the category of $A$-projective groups and $\mathscr{P}_{R}$ the category of projective right $R$-modules, where $R=\operatorname{End}(A)$. In view of the preceding remarks, it suffices to prove that: (i) $H$ sends $A$-free groups to free $R$ modules; (ii) $T$ sends free $R$-modules to $A$-free groups; and (iii) $\theta_{G}: T H(G)$ $\rightarrow G$ and $\phi_{M}: M \rightarrow H T(M)$ are isomorphisms for all objects $G$ and $M$ of $\mathscr{P}_{A}$ and $\mathscr{P}_{R}$, respectively.

It is easy to verify that $\theta_{A}: T H(A) \rightarrow A$ and $\phi_{R}: R \rightarrow H T(R)$ are isomorphisms (regard $R$ as a right $R$-module). Clearly $T$ preserves direct sums. Furthermore, $H\left(\Sigma \oplus G_{i}\right)=\operatorname{Hom}_{Z}\left(A, \Sigma \oplus G_{i}\right) \simeq \Sigma \oplus \operatorname{Hom}_{Z}\left(A, G_{i}\right)$ : since $A$ has finite rank, the image of $A$ under a homomorphism into $\Sigma \oplus G_{i}$ is contained in a subgroup generated by finitely many $G_{i}$. Consequently, if $G$ is $A$-free then $H(G)$ is a free $R$-module and if $M$ is a free $R$-module then $T(M)$ is $A$-free. In these two cases $\theta_{G}$ and $\phi_{M}$ are isomorphisms. Since $H$ and $T$ commute with direct sum decompositions, $\theta_{G}$ and $\phi_{M}$ are isomorphisms if $G$ is $A$-projective and $M$ is $R$-projective.

Remark. Warfield [16] proved Theorem 1.1 for the case that $\operatorname{rank}(A)=1$.

Corollary 1.2. Let $A$ be a torsion free abelian group of finite rank. Then (I) is true iff every projective right End $(A)$-module is free.

Call a set $\mathcal{F}=\left\{A_{i}\right\}_{i \in I}$ of abelian groups semirigid if there is a partial ordering on the index set, $l$, such that $i \leq j$ iff $\operatorname{Hom}_{Z}\left(A_{i}, A_{j}\right) \neq 0$. Define $\mathcal{F}_{\Sigma}$ to be the class of groups isomorphic to direct sums of groups in $\mathcal{F}$.

Corollary 1.3. Let $\mathcal{F}=\left\{A_{i}\right\}_{i \in l}$ be a semirigid set of torsion free abelian groups of finite rank such that (I) is true for all $A$ in $\mathfrak{F}$. Then any two direct sum decompositions of an $\mathfrak{F}_{\Sigma}$ group $G$ have isomorphic refinements. In particular, every summand of $G$ is an $\mathfrak{F}_{\Sigma}$ group.

Proof. Note that $G$ is a direct sum of countable groups. Thus if $B \oplus C$ $=G$ then $B$ is a direct sum of countable groups (e.g., see Fuchs [8, p. 49]). So assume that $B$ is a countable summand of $G$. Now $G=\sum_{i \in l} \bigoplus G(i)$, where $G(i)$ is either 0 or a direct sum of groups isomorphic to $A_{i}$. But $B=$ $\Sigma_{i \in I} \bigoplus B(i)$, where each $B(i)$ is either 0 or a summand of $G(i)$ (Charles [5]). 
By Corollary 1.2 , each $B(i)$ is an $A_{i}$-free group. The corollary now follows from the observation that two $A_{i}$-free groups are isomorphic iff they have the same cardinal number of summands isomorphic to $A_{i}$.

Remark. Corollary 1.3 includes the classical Baer-Kulikov-Kaplansky theorem (e.g., see Fuchs [9, p. 114]) and a theorem by Murley [14] as special cases.

2. Splitting and quasi-splitting exact sequences.

Theorem 2.1. Let $A$ be a finite rank torsion free abelian group. Then (II) is true iff $I A \neq A$ for all proper right ideals $I$ of End $(A)$.

Proof. $(\Leftarrow)$ It is sufficient to prove that every exact sequence $0 \rightarrow B$ $\rightarrow G \stackrel{\pi}{\rightarrow} A \rightarrow 0$ of abelian groups with $S_{A}(G)+B=G$ is split exact.

Define $I=\{\pi h \mid h: A \rightarrow G\}$, a right ideal of $R$. Then clearly $I A=A$. By assumption, $I=\operatorname{End}(A)$ so there is some $h: A \rightarrow G$ with $\pi h=1$. Consequently, (II) is true for $A$.

$(\Rightarrow$ ) Let $I$ be a right ideal of $R=\operatorname{End}(A)$ with $I A=A$ and choose a free right $R$-module $P$ with an epimorphism $\pi: P \rightarrow I$. The map $\mu: I \otimes_{R} A$ $\rightarrow A$ induced by $\mu(x \otimes a)=x a$ is epic since $I A=A$. Since $T(-)$ is right exact, $T(\pi): T(P) \rightarrow T(I)$ is epic so $\sigma=\mu T(\pi): P \otimes_{R} A \rightarrow A$ is epic. But $P$ $\otimes_{R} A$ is an abelian group with $S_{A}\left(P \otimes_{R} A\right)=P \otimes_{R} A$ so that $\sigma: P \otimes_{R} A$ $\rightarrow A$ splits. In particular, $H(\sigma): H T(P) \rightarrow H(A)$ is epic. Let $i: l \rightarrow H(A)$ be the inclusion map. Then $i \pi=H(\sigma) \phi_{P}$, where $\phi_{P}: P \rightarrow H T(P)$ is an isomorphism (Theorem 1.1). Consequently, $i$ is epic and $I=H(A)=R$.

Theorem 2.2. Suppose that $A$ is a finite rank torsion free abelian group and that either $A$ is strongly indecomposable, $Q \otimes_{Z}$ End $(A)$ is semisimple or End $(A)$ is commutative. If $I$ is a right ideal of $\operatorname{End}(A)$ with $I A=A$ then End $(A) / I$ is finite.

Proof. We prove that if $I A=A$ then $I$ contains a monomorphism $f$. Since monomorphisms are units in $Q \otimes_{Z}$ End $(A)$, there is a $g \in$ End $(A)$ with $f g=$ $n$ for some nonzero integer $n$. Thus $n$ End $(A) \subset I \subset$ End $(A)$ and $E n d(A) / I$ is finite (e.g., End $(A)$ is torsion free of finite rank so End $(A) / n$ End $(A)$ is bounded and contained in a finite direct sum of copies of $Q / Z$ ).

If $A$ is strongly indecomposable then every $0 \neq f \in \operatorname{End}(A)$ is monic or nilpotent (Reid [15]). Moreover, every nil ideal of End $(A)$ is nilpotent (since $Q \otimes_{Z}$ End $(A)$ is artinian) so $I$ must contain a monomorphism.

Assume that End $(A)$ is commutative and $I A=A$ for some ideal $I$ of End $(A)$. Then $Q \otimes_{Z} \operatorname{End}(A)$ is commutative, $Q \otimes_{Z} A$ is a finitely generated 
$Q \otimes_{Z}$ End $(A)$-module and $\left(Q \otimes_{Z} I\right)\left(Q \otimes_{Z} A\right)=Q \otimes_{Z} A$. Consequently, $(1+y)\left(Q \otimes_{Z} A\right)=0$ for some $y \in Q \otimes_{Z} I$ (Kaplansky [10, Theorem 76]). But this means that $y=-1$ and it follows that $I$ contains an integer.

Finally, if $Q \otimes_{Z}$ End $(A)$ is semisimple then $Q \otimes_{Z} I$ is principal and the theorem follows easily.

Corollary 2.3. Suppose that $A$ is a finite rank torsion free abelian group such that either $A$ is strongly indecomposable, $Q \otimes_{Z}$ End $(A)$ is semisimple or $\operatorname{End}(A)$ is commutative. If

$$
0 \rightarrow B \rightarrow G \stackrel{\pi}{\rightarrow} C \rightarrow 0
$$

is an exact sequence of torsion free abelian groups such that $S_{A}(G)+B=G$ and $C$ is a finite rank $A$-projective group then $(*)$ is a quasi-split sequence (i.e., there is $\delta: C \rightarrow G$ with $\pi \delta=n$ for some $0 \neq n \in Z$ ).

Proof. Let $C=A$ and $I=\left\{\pi h: h \in \operatorname{Hom}_{Z}(A, G)\right\}$, a right ideal of End $(A)$ with $I A=A$. By $2.2, n \operatorname{End}(A) \subseteq I \subseteq$ End $(A)$ for some $0 \neq n \in Z$. Consequently, $n=\pi \delta$ for some $\delta \in \operatorname{Hom}_{Z}(A, G)$. The corollary now follows in case $C$ is $A$-projective.

Corollary 2.4. If $A$ is a finite rank torsion free abelian group and if End $(A)$ is commutative or right principal then (II) is true for $A$.

Proof. By 2.1 it suffices to prove that if $I$ is a right ideal of $A$ with $I A$ $=A$ then $I=$ End $(A)=R$. If $R$ is right principal then $I=f R$ and $f(A)=A$ for some $f \in I$. Thus $f$ is a unit in $R$ and $I=R$.

Assume that $R$ is commutative. By Theorem $2.2, R /$ is finite, say $n R C$ $I \subset R$ for some $0 \neq n \in Z$. Let $\bar{R}=R / n R, \bar{l}=I / n R$ and $\bar{A}=A / n A$. Then $\bar{R}$ is commutative, $\bar{A}$ is a finitely generated $\bar{R}$-module and $\bar{I} \bar{A}=\bar{A}$. Once again, $(\overline{1}+\bar{y})(\bar{A})=0$ for some $y \in I$. Thus $(1+y)(A) \subseteq n A$ so that $1+y \in n R$ and $1 \in I+n R=I$. This proves that $R=I$.

Remark. If $\operatorname{End}(A)$ is a nonprincipal Dedekind domain then $A$ satisfies (II) but not (I). Corollary 2.4, for the case that $\operatorname{rank}(A)=1$, is a well-known theorem of Baer (Fuchs [9, p. 114]).

Example 2.5. Let $A=B \oplus C$ be a finite rank torsion free abelian group with $S_{B}(C)=C$ and $S_{C}(B)=0$ (e.g., $B=Z$ and $C$ has no free summands). Then there is a two-sided ideal $I$ of $\operatorname{End}(A)$ such that $I A=A$ and $\operatorname{End}(A) / I$ is infinite.

Proof. Let $I=\{f \in$ End $(A): f(C)=0\}$, a two-sided ideal of End $(A)$ since $C$ is fully invariant. Then $A=S_{B}(A)=I A$ and $Z \pi_{C} \subset$ End $(A) / I$ where $\pi_{C}$ : $A \rightarrow C$ is a projection map. 
Remark. The following question remains open: Is it true that if $A$ is strongly indecomposable and $I$ is a right ideal of $\operatorname{End}(A)$ with $I A=A$ then $I=\operatorname{End}(A)$ ? The answer is yes if $\operatorname{rank}(A) \leq 2($ see $\$ 5)$.

3. Subgroups of $A$-free groups. Let $\mathcal{S}_{A}$ be the category of subgroups $B$ of $A$-free groups with $S_{A}(B)=B$ and $\mathcal{S}_{R}$ the category of submodules of free right $R$-modules, where $R=\operatorname{End}(A)$. Note that $\delta_{A}$ is closed under direct sums and summands. We write $t(G)$ for the torsion subgroup of an abelian group $G$.

It is well known that if $A$ and $G$ are torsion free abelian groups then $G \otimes_{Z} A$ is torsion free. But if $G$ is a right $R$-module, where $R=\operatorname{End}(A)$, then $G \otimes_{R} A$ may not be torsion free (the examples are somewhat complicated, require that $\operatorname{End}(A)$ be a non-Dedekind domain such that $A$ is not a flat End( $A$ )-module and will be contained in a forthcoming paper).

Theorem 3.1. Assume that $A$ is a finite rank torsion free abelian group, $R=\operatorname{End}(A)$ and that $Q \otimes_{Z} R$ is semisimple. Then $U H$ is naturally equivalent to the identity functor on $\mathcal{S}_{A}$, where $H(-)=\operatorname{Hom}_{Z}(A,-)$ and $U(-)=$ $\left(-\otimes_{R} A\right) / t\left(-\otimes_{R} A\right)$.

Proof. Let $M$ be a submodule of the free right $R$-module $L$. Since $Q \otimes_{Z}$ $R$ is semisimple, $Q \otimes_{Z} A$ is a flat $Q \otimes_{Z} R$-module. The inclusion $i: M \rightarrow L$ induces a monomorphism $h: Q \otimes_{Z} M \otimes_{R} A \rightarrow Q \otimes_{Z} L \otimes_{R} A$. Moreover, there are monomorphisms $f: U(M) \rightarrow Q \otimes_{Z} M \otimes_{R} A$ and $g: U(L) \rightarrow Q \otimes_{Z} L \otimes_{R} A$ induced by $f(m \otimes a)=1 \otimes m \otimes a$ and $g(l \otimes a)=1 \otimes l \otimes a$, respectively. But $g i^{*}=h f$, where $i^{*}: U(M) \rightarrow U(L)$ is the map induced by $i$. Consequently, $i^{*}$ is monic and $U(M) \in \mathcal{S}_{A}$ since $U(L)$ is $A$-free (Theorem 1.1).

Let $B$ be a subgroup of an $A$-free group $G$ with $S_{A}(B)=B$. Then $\theta_{B}$ : $U H(B) \rightarrow B$ is epic (since $S_{A}(B)=B$ ). Furthermore, $\theta_{G} U H(i)=i \theta_{B}$ where $i: B \rightarrow G$ and $\theta_{B}$ is monic since $\theta_{G}$ is an isomorphism. Thus $U H$ is naturally equivalent to the identity functor on $\mathcal{S}_{A}$.

Remark. If $Q \otimes_{Z} \operatorname{End}(A)$ is semisimple then $A$ is a flat $\operatorname{End}(A)$-module iff the torsion subgroup of $I \otimes_{R} A$ is 0 for all finitely generated right ideals $I$ of $\operatorname{End}(A)=R$. In particular, if $\operatorname{End}(A)$ is right hereditary then $A$ is flat.

Corollary 3.2. Let $A$ be a finite rank torsion free abelian group.

(a) (II) and (III) are true iff $\operatorname{End}(A)$ is right principal with no zero-divisors.

(b) (II) and (IV) are true iff $\operatorname{End}(A)$ is right hereditary.

Proof. The proof of (a) is similar to the proof of (b).

(b) $(\Leftarrow)$ If $\operatorname{End}(A)$ is right hereditary then $Q \otimes_{Z} \operatorname{End}(A)$ is right hereditary and artinian, hence semisimple (a consequence of Nakayama's lemma). Thus (IV) is a consequence of Theorem 3.1. 
To prove (II) suppose that $I$ is a right ideal of $\operatorname{End}(A)$ with $I A=A$. Since $I$ is projective, $I \simeq H T(I)$. But $T(I) \simeq I A=A$ so $I \simeq \operatorname{End}(A)$, i.e., $I$ is right principal. Now apply Corollary 2.4 to see that (II) is true.

(b) $(\Rightarrow)$ Let $I$ be a nonzero right ideal of $R=\operatorname{End}(A)$ and choose a free $R$-module $P$ with an epimorphism $\Pi: P \rightarrow I$. Since $T(\Pi): T(P) \rightarrow T(I)$ is epic, there is an epimorphism $\sigma: T(P) \rightarrow I A$. By (IV), $I A$ is $A$-projective and, by (II), $\sigma$ is split. Thus $H(\sigma): H T(P) \rightarrow H(I A)$ is epic. Let $i: I \rightarrow H(I A)$ be the inclusion map. Since $i \Pi=H(\sigma) \phi_{P}$ and $\phi_{P}$ is an isomorphism, $i$ is epic and $I \simeq H(I A)$. But $I A$ is $A$-projective so $I$ is a projective right $R$-module.

Remark. Special cases of Corollary 3.2 include a classical theorem by Baer-Kolettis (see Fuchs [9, p. 114]) and a theorem of Murley [14].

4. Direct sum decompositions and cancellation. As a further application of the equivalence in Theorem 1.1, we prove an exchange theorem with some interesting applications.

Theorem 4.1. Let $G$ be a finite'rank torsion free abelian group and $G=$ $A \oplus K=B \oplus C$. If no quasi-summand of $A$ is quasi-isomorphic to any quasisummand of $K$ then there exist decompositions $B=B_{1} \oplus B_{2}, C=C_{1} \oplus C_{2}$ so that $G=B_{1} \oplus C_{1} \oplus K=A \oplus B_{2} \oplus C_{2}$.

Proof. Let $H=\operatorname{Hom}_{Z}(G,-), R=\operatorname{End}(G)$, and let $N$ be the nil radical of $R$. If $X$ and $Y$ are summands of $G$ then, by Theorem 1.1, $X \simeq Y$ iff $H(X)$ $\simeq H(Y)$. Also $H(X) \simeq H(Y)$ iff $\bar{X} \simeq \bar{Y}$ where $\bar{X}=H(X) / H(X) N$. (e.g., see Bass [3, p. 90]). Furthermore $\bar{X}$ and $\bar{Y}$ are finitely generated (in fact, principal) right ideals in $R / N$. Conversely, if $\bar{W}$ is any summand of $\bar{X}$ then there exists a summand $P$ of $H(X)$ such that $\bar{W}=P / P N$ (e.g., Bass [3, p. 88-90]). Thus $\bar{W}=H(W) / H(W) N$, where $W$ corresponds to $P$ via Theorem 1.1 (in fact, $W=P G)$.

Furthermore, $X$ and $Y$ are quasi-isomorphic iff $Q \otimes_{Z} \bar{X} \simeq Q \otimes_{Z} \bar{Y}$ as right ideals in $Q \otimes_{Z}(R / N):$ given an isomorphism $\phi: Q \otimes_{Z} \bar{X} \simeq Q \otimes_{Z} \bar{Y}$ with inverse $\psi$, there exists an integer $n \neq 0$ such that $n \phi(\bar{X}) \subseteq \bar{Y}$ and $n \psi(\bar{Y}) \subseteq \bar{X}$. Thus $n \phi$ and $n \psi$ induce maps between $H(X)$ and $H(Y)$ and so, via Theorem 1.1, there are maps $\phi^{\prime}: X \rightarrow Y$ and $\psi^{\prime}: Y \rightarrow X$ such that $\phi^{\prime} \psi^{\prime}$ $=n=\psi^{\prime} \phi^{\prime}$. Therefore, $X$ and $Y$ are quasi-isomorphic. The other direction is similar but easier.

Applying the preceding remarks to the decomposition $G=A \oplus K=B \oplus$ $C$ we get $\bar{G}=\bar{A}+\bar{K}=\bar{B}+\bar{C}$. Since $Q \otimes_{Z}(R / N)=Q \otimes_{Z} \bar{G}$ is a finite dimensional algebra with trivial nil radical, it is semisimple. The minimal quasi-summands of $A$ and $K$ correspond to the simple submodules of $Q \otimes_{Z} \bar{A}$ 
and $Q \otimes_{Z} \bar{K}$. Since any nontrivial homomorphism between simple modules is an isomorphism, it follows from the hypothesis that there are no nontrivial homomorphisms from $Q \otimes_{z} \bar{A}$ to $Q \otimes_{Z} \bar{K}$ or conversely, and consequently there are no nontrivial homomorphisms between $\bar{A}$ and $\bar{K}$ in either direction. Thus $\bar{A}$ and $\bar{K}$ are fully invariant submodules of $\bar{G}$, so that $\bar{G}=\bar{B}_{1} \oplus \bar{B}_{2}$ $\oplus \bar{C}_{1} \oplus \bar{C}_{2}$ with $\bar{B}_{1} \oplus \bar{B}_{2}=\bar{B}, \bar{C}_{1} \oplus \bar{C}_{2}=\bar{C}, \bar{B}_{1} \oplus \bar{C}_{1}=\bar{A}$, and $\bar{B}_{2} \oplus \bar{C}_{2}$ $=\bar{K}_{\text {. }}$ We can lift these decompositions of $\bar{B}$ and $\bar{C}$ to get $B=B_{1} \oplus B_{2}, C=$ $C_{1} \oplus C_{2}$ for suitable $B_{i}$ and $C_{i}$. Furthermore, $B_{1} \oplus C_{1} \simeq A_{2} B_{2} \oplus C_{2} \simeq$ $K$ and $H(G)=H\left(B_{1}\right)+H\left(C_{1}\right)+H(K)+N=H(A)+H\left(B_{2}\right)+H\left(C_{2}\right)+N$. By Nakayama's lemma, the $N$ in this equation can be omitted. Since, for any summand $X$ of $G$, we have $X=H(X) G$, it follows that $G=B_{1}+C_{1}+K=A$ $+B_{2}+C_{2}$. Adding up ranks guarantees that these sums must be direct.

As a special case, we have the following:

Corollary 4.2. If $A \oplus K \simeq A \oplus K_{1}$ and no quasi-summand of $A$ is quasiisomorphic to any quasi-summand of $K$ then $K \simeq K_{1}$.

Corollary 4.3. Let $F=\left\{A_{i}\right\}_{i \in I}$ be a set of mutally non-quasi-isomorphic strongly indecomposable finite rank groups. Let $F_{\Sigma}$ be the class of finite direct sums of groups in $F_{\Sigma}$ and let $G=B \oplus C \in F_{\Sigma}$. Then

(a) $B=\Sigma \oplus B(i)$, where each $B(i)$ is an $A_{i}$-projective group.

(b) If each $A_{i}$ has property (I) then $B$ is an $F_{\Sigma}$ group and $G$ has the Krull-Schmidt property.

In particular, Corollary 4.3 applies to the case $F=\mathcal{E}$, the class of finite rank indecomposable groups $A$ with the $Z / p Z$-dimension of $A / p A \leq 1$ for all primes $p$ [14]. It then follows that $E_{\Sigma}$ groups have the Krull-Schmidt property.

The same basic pattern can be used to prove a theorem slightly different from Theorem 4.1:

Theorem 4.4. If $G$ is a finite rank torsion free abelian group and $G=A$ $\oplus K=B \oplus C$, where no quasi-summand of $A$ is quasi-isomorphic to any quasisummand of $C$, then $G=A \oplus B_{1} \oplus C$ for some $B_{1} \subseteq B$.

Proof. Using the notation and techniques of Theorem 4.1, $\operatorname{Hom}_{R}(\bar{A}, \bar{C})=$ 0 , so that $\bar{A} \subseteq \bar{B}$. Thus $\bar{B}=\bar{A} \oplus \bar{B}_{1}$ and the conclusion follows as in Theorem 4.1.

The following theorem may be deduced from the recent proof by Lady [11] that every torsion free abelian group of finite rank has (up to isomorphism) at most a finite number of summands. This proof used some deep results of 
classical ring theory. On the other hand, the following arguments are group theoretic in nature.

Theorem 4.5. Let $A$ be a torsion free abelian group of finite rank such that $\operatorname{End}(A)$ has no zero-divisors and is right principal. Then for any torsion free finite rank abelian group $H$ there are, up to isomorphism, only finitely many groups $H_{1}$ such that $A \oplus H \simeq A \oplus H_{1}$.

Proof. Let $G=A \oplus H=A_{1} \oplus H_{1}$ with $A \simeq A_{1}$ and let $a$ and $\alpha_{1}$ be the projections of $G$ onto $A$ and $A_{1}$ with kernels $H$ and $H_{1}$, respectively.

Assume $A \cap H_{1} \neq 0$. Then the restriction of $\alpha_{1}$ to $A$ is not monic. But $A \simeq A_{1}$ and nonzero endomorphisms of $A$ are monic, so it follows that $a_{1}(A)=0$. Thus $A \subseteq H_{1}$ and $H_{1}=A \oplus D$ where $D=H \cap H_{1}$. Then $G=A_{1}$ $\oplus A \oplus D$, so that $H \simeq A_{1} \oplus D \simeq A \oplus D=H_{1}$. Similarly, we dispose of the case $A_{1} \cap H \neq 0$.

Now suppose $G=A \oplus H=A_{1} \oplus H_{1}$ with $A \simeq A_{1}, A \cap H_{1}=0, A_{1} \cap H=0$, and suppose that $H$ and $H_{1}$ are not isomorphic, Choose an $A$-free subgroup $K \subseteq H$ of maximal rank such that $K$ is a quasi-summand of $H$. Then there is a pure subgroup $C \subseteq H$ such that $K \oplus C$ has finite index in $H$. Since $A$ has property (III) and $\left(S_{A}(H)+C\right) / C$ is quasi-isomorphic to $H / C \simeq K$, $\left(S_{A}(H)+C\right) / C$ is $A$-free. By property (II) there is an $A$-free subgroup $B$ of $H$ with $S_{A}(H)+C=B \oplus C$ (and $B \simeq K$ ). Then $S_{A}(H)=B \oplus S_{A}(C)$ (since $S_{A}$ commutes with direct sums).

Let $\eta$ and $\eta_{1}$ be the projections of $G$ onto $H$ and $H_{1}$ with kernels $A$ and $A_{1}$, respectively. Since $A \cap H_{1}=A_{1} \cap H=0, \eta$ and $\eta_{1}$ are monomorphisms and $\eta_{1}(H)$ has finite index in $H_{1}$. Now $\eta_{1}(B)$ is $A$-free, $\eta_{1}(B) \cap$ $C_{1}=0$ and $\eta_{1}(B) \oplus C_{1}$ has finite index in $H_{1}$, where $C_{1}$ is the pure subgroup of $H_{1}$ generated by $\eta_{1}(C)$. The previous argument now applies to give an $A$-free subgroup $B_{1}$ of $S_{A}\left(H_{1}\right)$ with $S_{A}\left(H_{1}\right)=B_{1} \oplus S_{A}\left(C_{1}\right)$. Necessarily, $B_{1} \simeq B$.

There is a nonzero integer $n$ such that $n H \subseteq B \oplus C \subseteq H$. Now $n H_{1}=$ $n \eta_{1}(G)=\eta_{1}(n A \oplus n H) \subseteq \eta_{1}(n A \oplus B \oplus C) \subseteq S_{A}\left(H_{1}\right)+\eta_{1}(C)$. If $S_{A}\left(H_{1}\right)+$ $\eta_{1}(C)=B_{1} \oplus \eta_{1}(C)$ then the proof would be complete. For $n$ is independent of $H_{1}$, and $B_{1} \oplus \eta_{1}(C) \simeq B \oplus C$, so $H_{1} \simeq H^{\prime}$ where $n(B \oplus C) \subseteq H^{\prime} \subseteq B \oplus$ C. Since $(B \oplus C) / n(B \oplus C)$ is a finite group, there are only finitely many such $H^{\prime}$.

To show that $S_{A}\left(H_{1}\right)+\eta_{1}(C)=B_{1} \oplus \eta_{1}(C)$, we need to show that $S_{A}\left(H_{1}\right)$ $\subseteq B_{1} \oplus \eta_{1}(C)$. Since $S_{A}\left(H_{1}\right)=B_{1} \oplus S_{A}\left(C_{1}\right)$, this amounts to showing that 
$S_{A}\left(C_{1}\right) \subseteq \eta_{1}(C)$. We claim first that $S_{A}\left(C_{1}\right) \subseteq H$. Otherwise there is a map $f: A \rightarrow C_{1}$ such that $a f \neq 0$ and $a f$ is a monomorphism (since $a f: A \rightarrow A$ ). It readily follows that $f(A)$ is a quasi-summand of $C_{1}$, so that $B \oplus$ af $(A)$ is an $A$-free quasi-summand of $H$, contradicting the maximality of $K$. Thus $S_{A}\left(C_{1}\right) \subseteq H$, and so $S_{A}\left(C_{1}\right) \subseteq S_{A}(H) \subseteq B \oplus C$ and $S_{A}\left(C_{1}\right)=\eta_{1}\left(S_{A}\left(C_{1}\right)\right) \subseteq$ $\left(\eta_{1}(B) \oplus \eta_{1}(C)\right) \cap C_{1}=\eta_{1}(C)$. This completes the proof.

Fuchs-Loonstra $[9, \mathrm{p} .138]$ showed that for any $n \geq 2$ there is a torsion free abelian group $G$ of rank 3 such that $G=A_{i} \oplus H_{i}, 1 \leq i \leq n$ where $\left\{A_{1}\right.$, $\left.\ldots, A_{n}\right\}$ is a set of isomorphic groups of rank $1 ; H_{i} \approx H_{j}$ iff $i=j$; and each $H_{i}$ is quasi-isomorphic to $A_{i} \oplus B$ for some rank 1 group $B$ such that type $\left(A_{i}\right)$ and type $(B)$ are incomparable. The following corollary indicates that these examples were well chosen.

Corollary 4.6 (J. Cook [6]). Assume that $G$ is a torsion free abelian group of rank 3.

(a) Suppose that $G=A_{1} \oplus H_{1}=A_{2} \oplus H_{2}$, where $A_{1}$ and $A_{2}$ are rank 1 groups, and that $H_{1}$ and $H_{2}$ are not isomorphic. Then $A_{1}$ and $A_{2}$ are isomorphic and $H_{2}$ (hence $H_{1}$ ) is quasi-isomorphic to $A_{1} \oplus B_{1}$, where $B_{1}$ is a rank 1 group such that type $\left(A_{1}\right)$ and type $\left(B_{1}\right)$ are not comparable.

(b) $G$ has at most a finite number of nonequivalent direct sum decompositions.

Proof. (a) By Corollary 4.2, $H_{2}$ must be quasi-isomorphic to $A_{1} \oplus B_{1}$ for some $B_{1}$. If type $\left(A_{1}\right)$ and type $\left(B_{1}\right)$ are comparable, then $H_{2} \simeq A_{1} \oplus$ $B_{1}$ (Beaumont-Pierce [4]) so that $G$ is completely decomposable and $H_{1} \simeq$ $H_{2}$, contradicting the assumptions. Consequently, type $\left(A_{1}\right)$ and type $\left(B_{1}\right)$ are not comparable. As in the proof of Theorem 4.5, $A_{1} \cap H_{2}=A_{2} \cap H_{1}=0$, so it follows that $A_{1}$ and $A_{2}$ are quasi-isomorphic, hence isomorphic, since both $A_{1}$ and $A_{2}$ are rank 1 groups.

(b) is now a consequence of (a) and Theorem 4.5.

5. Groups of rank 2. A torsion free abelian group is homogeneous if any two pure rank 1 subgroups are isomorphic.

Corollary 5.1. Let $A$ be a strongly indecomposable torsion free abelian group of rank 2.

(a) (II) is true for $A$.

(b) If $A$ is not homogeneous then every finite rank A-projective group is $A \cdot f r e e$. 
Proof. (a) In this case, $\operatorname{End}(A)$ is commutative (e.g., Beaumont-Pierce [4] or Reid [15]), so Corollary 2.4 applies.

(b) It is known that $Q \otimes_{Z} \operatorname{End}(A)$ is either $Q$ or the ring of $2 \times 2$ triangular matrices over $Q$ with equal diagonal elements (see Beaumont-Pierce [4] or Reid [15]). In the first case, $\operatorname{End}(A)$ is a principal ideal domain, so (I) is true by Corollary 1.2.

In the second case $S=\operatorname{End}(A) / N$ is a subring of $Q$ and thus is a principal ideal domain, where $N$ is the nil radical. Thus finitely generated projective $S$-modules are free so finitely generated projective $\operatorname{End}(A)$-modules are free (see proof of Theorem 4.1). Now apply Corollary 1.2.

Theorem 5.2. Let $R$ be a nonprincipal ring of integers in a quadratic algebraic number field, and let $I$ be a nonprincipal ideal in $R$. Then there exists a strongly indecomposable rank 2 group $A$ such that $\operatorname{End}(A) \simeq R$. The subgroup IA is a summand of $A \oplus A$ but is not A-free. If $n$ is the order of $I$ in the ideal class group of $R$, then [A]- [IA] is an element of order exactly $n$ in the Krull-Schmidt-Grothendieck group of the category of finite rank torsion free abelian groups.

Proof. There exists a rank 2 group $A$ with $\operatorname{End}(A) \simeq R$ by [17]. Since $R$ is an integral domain, $A$ is strongly indecomposable. By Theorem 1.1, $B$ $=I A$ is $A$-projective. (Note that $I A \simeq I \otimes_{R} A$, since $I$ is projective.) Also $B \oplus \cdots \oplus B \simeq A \oplus \ldots \oplus A$ ( $n$ summands), since $I \oplus \cdots \oplus I \simeq R \oplus \cdots$ $\oplus R$ ( $n$ summands). Now we claim that $[A]$ and $[B]$ are distinct elements of the Krull-Schmidt-Grothendieck group. Otherwise, it would be true that $A \oplus L \simeq B \oplus L$ for some finite rank torsion free group $L$. Applying $\operatorname{Hom}_{Z}(A, *)$ yields an isomorphism $R \oplus M \simeq I \oplus M$ where $M$ is a finite rank torsion free $R$-module. Now write $M=P \oplus S$, where $P$ is projective and $S$ has no projective summands. Since $R$ is a Dedekind domain, this implies that $\operatorname{Hom}_{R}(S, I \oplus P)=0$, so the above isomorphism maps $S$ onto itself and thus induces an isomorphism between $R \oplus P$ and $I \oplus P$. Since $R$ and $I$ represent different elements in the ideal class group, this is in contradiction to the well-known structure theory for finitely generated projective modules over Dedekind domains. Thus we have shown that $[A]-[B]$ is a nontrivial element in the Krull-Schmidt-Grothendieck group with order dividing $n$. Now if $r$ is a proper divisor of $n$ then $I \oplus \cdots \oplus I$ ( $r$ summands) is isomorphic to $R \oplus \ldots \oplus R \oplus I^{r}$. It follows that $B \oplus \cdots \oplus B \simeq A \oplus \cdots \oplus A \oplus I^{r} A$. Since the argument above shows that $\left[I^{r} A\right] \neq[A]$, we see that $r([A]-[B]) \neq 0$. 
By using a theorem of Zassenhaus [17] or the well-known theorem of Corner [7], we can construct such examples where $R$ is any algebraic number ring. It follows that the Krull-Schmidt-Grothendieck group for the category of finite rank torsion free abelian groups contains a copy of the class group of every algebraic number field (as announced by Arnold [1]).

Example 5.3. There is a homogeneous strongly indecomposable group $A$ of rank 2 and a subgroup $B$ of $A$ such that $S_{A}(B)=B, A / B$ is finite and $B$ is not $A$-projective (in particular, $B$ and $A$ are not isomorphic). Furthermore, every finite rank $A$-projective group is $A$-free.

Proof. Let $R=Z+2 Z i$, a subring of the ring of Gaussian integers. By Zassenhaus [17], there is a rank 2 group $A$ with $\operatorname{End}(A)=R$. Let $I$ be the ideal of $R$ generated by $\{2,2 i\}$, a nonprincipal nonprojective ideal of $R$. Then $B=I A$ has the desired properties. The latter statement follows from the observation that every invertible ideal of $R$ is principal and every finitely generated projective $R$-module is a direct sum of ideals of $R$.

\section{REFERENCES}

1. D. M. Arnold, Grothendieck and Whitehead groups of torsion free abelian groups, Bull. Amer. Math. Soc. 79 (1973), 723-724. MR 48 \#4149.

2. R. Baer, Abelian groups without elements of finite order, Duke Math. J. 3 (1937), 68-122.

3. H. Bass, Algebraic K-theory, Benjamin, New York, 1968. MR $40 \# 2736$.

4. R. A. Beaumont and R.S. Pierce, Torsion free groups of rank two, Mem. Amer. Math. Soc. No. 38 (1961). MR 24 \#A162.

5. B. Charles, Sous-groupes fonctoriels et topologies, Studies in Abelian Groups (Sympos., Montpellier, 1967), Springer, Berlin, 1968, pp. 75-92. MR 39 \#1547.

6. J. Cook, Thesis, Univ. of Illinois, 1971.

7. A. L. S. Corner, Every countable reduced torsion-free ring is an endomorphism ring, Proc. London Math. Soc. (3) 13 (1963), 687-710. MR 27 \#3704.

8. L. Fuchs, Infinite abelian groups. Vol. I, Pure and Appl. Math., vol. 36, Academic Press, New York and London, 1970. MR 41 \#333.

9. - Infinite Abelian groups. Vol. II, Academic Press, New York, 1973.

10. I. Kaplansky, Commutative rings, Allyn and Bacon, Boston, Mass., 1970. MR 40 \#7234.

11. L. Lady, Summands of finite rank torsion free abelian groups, J. Algebra 32 (1974), 51-52.

12. J. Lambek, Lectures on rings and modules, Blaisdell, Waltham, Mass., 1966. MR 34 \#5857.

13. C. E. Murley, The classification of certain classes of torsion free abelian groups, Pacific J. Math. 40 (1972), 647-665. MR 48 \#441.

14. - Direct products and sums of torsion-free abelian groups, Proc. Amer. Math. Soc. 38 (1973), 235-241. MR 47 \#362. 
15. J.D. Reid, On quasi-decomposition of torsion free abelian groups, Proc. Amer. Math. Soc. 13 (1962), 550-554. MR 25 \#1209.

16. R. B. Warfield, Jr., Homomorphisms and duality of torsion-free groups, Math. Z. 107 (1968), 189-200. MR 38 \#5923.

17. H. J. Zassenhaus, Orders as endomorphism rings of modules of the same rank, J. London Math. Soc. 42 (1967), 180-182. MR 34 \#5876.

DEPARTMENT OF MATHEMATICAL SCIENCES, NEW MEXICO STATE UNIVERSITY, LAS CRUCES, NEW MEXICO 88003

DEPARTMENT OF MATHEMATICS, UNIVERSITY OF KANSAS, LAWRENCE, KANSAS 66045 